\title{
D'ALEMBERT'S FUNCTIONAL EQUATION ON GROUPS
}

\author{
R. C. PENNEY ${ }^{1}$ AND A. L. RUKHIN ${ }^{2}$
}

ABSTRACT. Group representation theory is used to solve a functional equation of the D'Alembert type on groups.

I. Reduction to a representation theory problem. We consider a functional equation of the form

$$
\phi_{1}(g h)+\phi_{2}\left(g^{-1} h\right)=\sum_{i=1}^{n} \kappa_{i}(g) \lambda_{i}(h)
$$

where $\phi_{1}, \phi_{2}, \kappa_{i}, \lambda_{i}, i=1, \ldots, n$, are some (measurable) complex functions given on a locally compact group $G$. We can and shall assume that functions $\kappa_{i}$ and $\lambda_{i}, i=1, \ldots, n$, are linearly independent. The equation (*) can be viewed as a generalization of the known D'Alembert functional equation

$$
\phi(g h)+\phi\left(g^{-1} h\right)=2 \phi(g) \phi(h),
$$

which has been studied by many authors (cf. [4]-[6]). A particular case of (*) when $\phi_{1}=-\phi_{2}$ arises in some statistical applications and was studied by one of the authors in the situation when $G$ is a compact Lie group [7]. The solution of (*) in the case $G=R^{1}$ can be found in Aczél's book [1, pp. 171-176, 199].

In this paper we present the solution of (*) in the case when

$$
\int\left|\phi_{i}(g)\right|^{2} d \nu(g)<\infty, \quad i=1,2,
$$

where $\nu$ is the left Haar measure on $G$. Henceforth we denote the space of such functions by $L_{2}(G)$. We also consider the case when $\phi_{i}, i=1,2$, can be represented as a finite combination of positive definite functions.

Note that if $\varphi(g)=\phi_{1}(g)+\phi_{2}(g), \xi(g)=\phi_{1}(g)-\phi_{2}(g)$ then

$$
\begin{aligned}
\varphi(g h)+\varphi\left(g^{-1} h\right) & =\sum_{i=1}^{n}\left[\kappa_{i}(g)+\kappa_{i}\left(g^{-1}\right)\right] \lambda_{i}(h) \\
& =\sum_{i=1}^{m} \alpha_{i}(g) \varphi_{i}(h)
\end{aligned}
$$

Received by the editors November 9, 1978, and, in revised form, December 19, 1978.

AMS (MOS) subject classifications (1970). Primary 39A40; Secondary 22D12.

${ }^{1}$ This research has been supported by NSF Grant 7804898.

${ }^{2}$ This research has been supported by NSF Grant \# MCS 77019640. 
and

$$
\begin{aligned}
\xi(g h)-\xi\left(g^{-1} h\right) & =\sum_{j=1}^{n}\left[\kappa_{i}(g)-\kappa_{j}\left(g^{-1}\right)\right] \lambda_{j}(h) \\
& =\sum_{j=1}^{r} \beta_{j}(g) \xi_{j}(h)
\end{aligned}
$$

with linearly independent functions $\alpha_{i}, \varphi_{i}, i=1, \ldots, m$, and $\beta_{j}, \xi_{j}, j=$ $1, \ldots, r$. Therefore we restrict our attention to the case $\phi_{1}=\phi_{2}$ or $\phi_{1}=$ $-\phi_{2}$ in $(*)$.

We reduce the solution of $(*)$ to a certain problem in representation theory in the following way. Let us begin with the equation (**). If $H$ denotes the closure in $L_{2}(G)$ of the linear space spanned by the left shifts $\varphi(g \cdot), g \in G$, of the function $\varphi$ then the left regular representation $U$ acts in $H: U(g) \eta(\cdot)$ $=\eta\left(g^{-1} \cdot\right), \eta \in H$, and $U(g)$ is a unitary operator. The relation (**) implies that

$$
\left[U(g)+U\left(g^{-1}\right)\right] \varphi=\sum_{i=1}^{m} \alpha_{i}(g) \varphi_{i}
$$

where $\varphi$ denotes the vector of $L$ corresponding to the function $\varphi(\cdot)$, and $\varphi_{1}, \ldots, \varphi_{m}$ are vectors from $H$. Because of the definition of $H, U$ is a cyclic representation with a cyclic vector $\varphi$ (i.e., the space spanned by vectors $U(g) \varphi, g \in G$, is dense in $H$ ). The following result is a corollary of these considerations and Theorem 2.1 .

THEOREM 1.1. Let $G$ be a locally compact group of type one such that the elements of the form $g^{4}, g \in G$, generate a dense subgroup of $G$. Every solution $\varphi \in L_{2}(G)$ of the equation (**) has the form

$$
\varphi(g)=\left\langle U\left(g^{-1}\right) \varphi, \eta\right\rangle
$$

where $U$ is a finite dimensional, unitary, cyclic (with a cyclic vector $\varphi$ ) representation of $G$ such that the space spanned by vectors $\left[U(g)+U\left(g^{-1}\right)\right] \varphi$, $g \in G$ has dimension $m$, and $\eta$ is some vector of the representation space $U$.

Note that a formula for the dimension of the representation $U$ can be obtained from Theorem 2.1.

It is immediately seen that the functional equation $(* * *)$ is equivalent to the finite dimensionality of the space spanned by vectors $\left[U(g)-U\left(g^{-1}\right)\right] \xi$, $g \in G$, where again $U$ is a unitary representation with a cyclic vector $\xi$.

THEOREM 1.2. Let $G$ be a locally compact group of type one such that the elements of the form $g^{2}, g \in G$, generate a dense subgroup of $G$. Every solution $\xi \in L_{2}(G)$ has the same form as indicated in Theorem 1.1 with $\xi$ instead of $\varphi$, $\left[U(g)-U\left(g^{-1}\right)\right] \xi$ instead of $\left[U(g)+U\left(g^{-1}\right)\right] \varphi$ and $r$ instead of $m$.

The solution of the general equation (*) now follows easily from Theorems 1.1 and 1.2 . 
The content of these theorems is that if $G$ is noncompact then the D'Alembert functional equation has few solutions, as noncompact groups usually have few finite dimensional unitary representations.

THEOREM 1.3. Under the assumptions of Theorem 1.1, if there exists a nonzero solution of (**) then $G$ is compact.

Proof. It follows from Theorem 1.1 that every solution of $(* *)$ has the form

$$
\varphi(g)=\left\langle U\left(g^{-1}\right) \varphi, \eta\right\rangle
$$

with a finite dimensional representation $U$. However, such a matrix element cannot be square integrable unless $G$ is compact. Indeed let $K$ be the kernel of $U$. Clearly $K$ is a closed, normal subgroup of $G$ and $\varphi$ is constant on cosets of $K$ in $G$. In order for $\varphi$ to be square integrable, $K$ must have finite volume with respect to Haar measure which implies the compactness of $K$.

To prove that $G$ is compact, it suffices to show that $G / K$ is compact. To this end we may assume that $K=\{e\}$ so that $U$ is injective. But then $G$ is compactly injectible, and hence $C$ is the product of a compact group and $R^{p}$ for some $p$ (cf. $[2, \S 16.4 .2]$ ). But $R^{p}$ has no injective, finite dimensional unitary representations unless $p=0$. Thus $G$ is compact.

We give another version of Theorem 1.1.

THEOREM 1.4. Theorem 1.1 holds if one assumes that $\varphi$ is a linear combination of positive definite functions instead of $\varphi \in L_{2}(G)$.

Proof. It follows from Godement [3] that there exists a unitary representation $U$ and vectors $\varphi$ and $\eta$ such that

$$
\varphi(g)=\left\langle U\left(g^{-1}\right) \varphi, \eta\right\rangle .
$$

These vectors $\varphi$ and $\eta$ can be assumed to be cyclic for $U$ (the latter since $\varphi(g)=\langle\varphi, U(g) \eta\rangle)$. Moreover we can replace $\eta$ by its projection onto the closed subspace spanned by the vectors $U\left(g^{-1}\right) \varphi$. Because of (**) the space of functions $\left\langle\left[U(g)+U\left(g^{-1}\right)\right] \varphi, U(\cdot) \eta\right\rangle, g \in G$, is finite dimensional. The cyclicity of $\eta$ implies that the space spanned by the vectors $[U(g)+$ $\left.U\left(g^{-1}\right)\right] \varphi$ is finite dimensional and the cyclicity of $\varphi$ and Theorem 2.1 imply that $U$ is finite dimensional.

2. Symmetric and antisymmetric intertwining operators. Let $U$ be a unitary representation of the topological group $G$ in a Hilbert space $H$. Let $H^{*}$ be the continuous dual of $H$ and let $U^{*}$ be the contragradient representation to $U$, i.e.,

$$
\left\langle\xi, U^{*}(g) \eta\right\rangle=\left\langle U\left(g^{-1}\right) \xi, \eta\right\rangle
$$

Clearly $H^{*}$ is a Hilbert space which is conjugate isomorphic to $H$ and $U^{*}$ is a unitary operator.

Let $I\left(U, U^{*}\right)$ denote the set of all continuous operators $A$ mapping $H$ into 
$U^{*}$ such that

$$
U^{*}(g) A=A U(g) .
$$

If $A^{*}$ is the dual operator, $A^{*}: H^{* *}=H \rightarrow H^{*}$, then $A$ is said to be symmetric if $A^{*}=A$ and antisymmetric if $A^{*}=-A$. The space of symmetric elements of $I\left(U, U^{*}\right)$ is denoted $I_{s}\left(U, U^{*}\right)$ and the space of antisymmetric elements is denoted $I_{a}\left(U, U^{*}\right)$. Clearly

$$
I\left(U, U^{*}\right)=I_{s}\left(U, U^{*}\right)+I_{a}\left(U, U^{*}\right) .
$$

Note that $A^{*}$ is not the same as the Hilbert space adjoint of $A$ which is a mapping of $H^{*}$ to $H$.

We prove the following.

THEOREM 2.1. Let $G$ be a locally compact group and $U$ a type one unitary representation of $G$ which possesses a cyclic vector $\varphi$. Let $L_{+}$and $L_{-}$be the subspaces of $H$ defined as

$$
L_{ \pm}=\operatorname{span}\left\{\left[U(g) \pm U\left(g^{-1}\right)\right] \varphi, g \in G\right\} .
$$

Then:

(a) if $L_{-}$is finite dimensional and the elements of the form $g^{2}, g \in G$, generate $a$ dense subgroup of $G$, then $U$ is finite dimensional and

$$
\operatorname{dim} U=\operatorname{dim} L_{-}+\operatorname{dim} I_{s}\left(U, U^{*}\right) \text {; }
$$

(b) if $L_{+}$is finite dimensional and the elements of the form $g^{4}, g \in G$, generate a dense subgroup of $G$ then $U$ is finite dimensional and

$$
\operatorname{dim} U=\operatorname{dim} L_{+}+\operatorname{dim} I_{a}\left(U, U^{*}\right) .
$$

Proof. Since the proofs of (a) and (b) are similar we prove only (b). Let $L^{\prime}$ be the annihilator of $L_{+}$in $H^{*}$. We shall establish a one-to-one correspondence between $L^{\prime}$ and $I_{a}\left(U, U^{*}\right)$. Specifically, the correspondence will be obtained as follows. Let $\lambda \in L^{\prime}$. For each vector of the form

define

$$
\xi=\sum_{i} c_{i} U\left(g_{i}\right) \varphi
$$

$$
B_{\lambda}(\xi)=\sum_{i} c_{i} U^{*}\left(g_{i}\right) \lambda .
$$

We shall show that this correspondence is well defined. Granting this, $\boldsymbol{B}_{\boldsymbol{\lambda}}$ becomes a densely defined intertwining operator from $H$ to $H^{*}$. The main problem in proving our theorem is to demonstrate that $B_{\lambda}$ is, in fact, a bounded operator. This will be achieved by expanding the domain of $B$ as much as possible.

Now, let $V=U \oplus U^{*}$, and let $\mathscr{Q}$ be the von Neumann algebra on $H \oplus H^{*}$ generated by the set of operators $\{V(g), g \in G\}$. Each element of $Q$ is an operator of the form $A \oplus A^{\prime}$ where

$$
A=\lim _{\alpha} \sum_{g \in G} c^{\alpha}(g) U(g), \quad A^{\prime}=\lim _{\alpha} \sum_{g \in G} c^{\alpha}(g) U^{*}(g),
$$


and $c^{\alpha}$ is a net of functions on $G$ which are supported on finite sets on $G$. The limits are taken in the strong operator topology.

Lemma 2.1. Suppose $A \oplus A^{\prime} \in \mathbb{Q}$ and $\lambda \in L^{\prime}$. Then $A \varphi=0$ implies that $A^{\prime} \lambda=0$.

Proof. Let $A$ and $A^{\prime}$ be represented as described above and consider the function

$$
f(h)=\left\langle U(h) \varphi, A^{\prime} \lambda\right\rangle
$$

By definition

$$
f(h)=\lim _{\alpha} \sum_{g \in G}\left\langle U(h) \varphi, c^{\alpha}(g) U^{*}(g) \lambda\right\rangle=\lim _{\alpha} \sum_{g \in G}\left\langle U\left(g^{-1} h\right) \varphi, \lambda\right\rangle c^{\alpha}(g) .
$$

Since $\lambda \in L^{\prime}$,

$$
\langle U(g) \varphi, \lambda\rangle=-\left\langle U\left(g^{-1}\right) \varphi, \lambda\right\rangle .
$$

Thus

$$
\begin{aligned}
f(h) & =\lim _{\alpha} \sum_{g \in G}\left\langle U\left(h^{-1}\right) c_{\alpha}(g) U(g) \varphi, \lambda\right\rangle \\
& =\left\langle U\left(h^{-1}\right) A \varphi, \lambda\right\rangle=0
\end{aligned}
$$

and $A^{\prime} \lambda=0$ because of the cyclicity of $\varphi$. The lemma is proven.

Let $\mathcal{C}$ be the subspace of $H$ defined by $\mathcal{C}=\left\{A \varphi: A \oplus A^{\prime} \in \mathbb{Q}\right.$ for some $\left.A^{\prime}\right\}$. For each $\lambda \in L^{\prime}$ let $B_{\lambda}$ be the mapping of $\mathcal{C}$ into $H^{*}$ defined as $B_{\lambda}(A \phi)=A^{\prime} \lambda$ where $A \oplus A^{\prime} \in \mathbb{Q}$. By Lemma 2.1 this definition makes sense.

LEMMA 2.2. For all $v, w \in \mathcal{C}$,

(i) $\left\langle v, B_{\lambda} w\right\rangle=-\left\langle w, B_{\lambda} v\right\rangle$, and

(ii) $B_{\lambda} U(g) v=U^{*}(g) B_{\lambda} v$.

Conversely, any linear operator $B: C \rightarrow H^{*}$ which satisfies (i) and (ii) has the form $B=B_{\lambda}$ where $\lambda=B \varphi \in L^{\prime}$.

Proof. The proof of (i) is analogous to that of Lemma 2.1 and (ii) follows from the definition of $B_{\lambda}$. The last statement of Lemma 2.2 is true since

$$
\begin{aligned}
\langle U(g) \varphi, B \varphi\rangle & =-\langle\varphi, B U(g) \varphi\rangle=-\left\langle\varphi, U^{*}(g) B \varphi\right\rangle \\
& =\left\langle-U\left(g^{-1}\right) \varphi, B \varphi\right\rangle
\end{aligned}
$$

Now let $\pi: H \rightarrow H$ be a central projection for $U$, i.e. $\pi$ commutes with $U$ and with the commuting algebra of $U$. We call $\pi$ balanced if $\pi \oplus \pi^{*}$ is a central projection for $V$. More specifically, the general "matrix" form of an

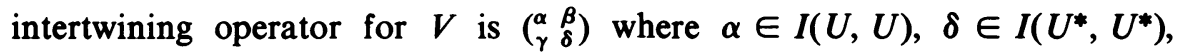
$\beta \in I\left(U^{*}, U\right)$ and $\gamma \in I\left(U, U^{*}\right)$. Hence $\pi$ is balanced if $\pi$ is central for $U$ and $\gamma \pi=\pi^{*} \gamma$ for all $\gamma \in I\left(U, U^{*}\right)$. (The $\beta$-identity follows from the $\gamma$-identity by transposition.) Intuitively, "balanced central projection" means that if an irreducible representation $U_{0}$ "occurs" in $\pi$, then $U_{0}^{*}$ also "occurs" in $\pi$ 
given that $U_{0}^{*}$ "occurs" in $U$. Of course, $U$ might have no discrete spectrum so this is only formal.

LEMMA 2.3. If $\pi$ is balanced, then $\pi L_{+} \subset L_{+}$. If there is a nonzero balanced projection $\pi$ such that $\pi L_{+}=0$ then the image of $\pi$ is one-dimensional and $U$ is trivial on the image of $\pi$.

Proof. It suffices to show that $\pi^{*}$ maps $L^{\prime}$ into $L^{\prime}$. Let $\lambda \in L^{\prime}$. Then $\lambda=B_{\lambda} \varphi$ and $\pi^{*} \lambda=\pi^{*} B_{\lambda} \varphi$. But then

$$
\begin{aligned}
\left\langle U(g) \varphi, \pi^{*} \lambda\right\rangle & =\left\langle U(g) \varphi, \pi^{*} B_{\lambda} \varphi\right\rangle=-\left\langle\varphi, B_{\lambda} \pi U(g) \varphi\right\rangle \\
& =-\left\langle\varphi, \pi^{*} U^{*}(g) \lambda\right\rangle=-\left\langle U\left(g^{-1}\right) \varphi, \pi^{*} \lambda\right\rangle
\end{aligned}
$$

since $\pi U(g) \oplus \pi^{*} U^{*}(g) \in \mathbb{Q}$. Thus, as claimed, $\pi^{*} \lambda \in L^{\prime}$.

Now, if $\pi\left(U(g)+U\left(g^{-1}\right)\right) \varphi=0$ then $U(g) \pi \varphi=-U\left(g^{-1}\right) \pi \varphi$, so that $U\left(g^{4}\right) \pi \varphi=\pi \varphi$. Since the elements $g^{4}$ generate a dense subgroup, $U$ is trivial on the image of $\pi$. Since a cyclic representation can contain the identity representation at most once, our lemma follows.

COROLlARY. There is only a finite number of disjoint balanced projections for U. Also $\sum_{i}^{q} \pi_{i}=1$ if $\pi_{1}, \ldots, \pi_{q}$ is a maximal family of disjoint balanced projections.

Proof. Let $\pi_{1}$ be the unique balanced projection such that $\pi_{1} L_{+}=0$ (it is possible that $\pi_{1}=0$ ). If $\left\{\pi_{i}\right\}$ is any family of disjoint balanced projections, then $\Sigma \bigoplus \pi_{i} L_{+} \subset L_{+}$. By finite dimensionality there can be at most a finite number of such $\pi_{i}$. The equality $\sum_{1}^{q} \pi_{i}=I$ for a maximal family follows from the fact that $\pi$ is balanced iff $I-\pi$ is balanced.

This corollary allows us to assume that in the proof of finite dimensionality of $U$, the identity is the only nonzero balanced projection. In this case $U$ is "almost" a primary representation as the next lemma shows.

LEMMA 2.4. $U$ has at most two disjoint central projections.

Proof. Let $\pi$ be a central projection for $U$. Let $H_{\pi}$ be the image of $\pi$ in $H$ and let $H_{\tilde{\pi}}$ be the closure of the image of $H_{\pi}$ under $I\left(U, U^{*}\right)$. Let $\tilde{\pi}$ be the projection in $H^{*}$ onto $H_{\tilde{\pi}}$. It is clear that $H_{\tilde{\pi}}$ is invariant under $U^{*}$ so that $\pi \oplus \tilde{\pi} \in \mathbb{Q}^{\prime}$. We claim also that $\pi \oplus \tilde{\pi} \in \mathbb{Q}$. To see this, it is sufficient to prove that $\pi \oplus \tilde{\pi}$ commutes with $\mathbb{Q}^{\prime}$. It is obvious that $H_{\tilde{\pi}}$ is invariant under any operator which commutes with $U^{*}$, so it suffices so show that $\tilde{\pi} A=A \pi$ for all $A \in I\left(U, U^{*}\right)$ and $\pi A^{\prime}=A^{\prime} \tilde{\pi}$ for all $A^{\prime} \in I\left(U^{*}, U\right)$. The first identity is proven as follows:

$$
\tilde{\pi} A=\tilde{\pi} A \pi+\tilde{\pi} A(I-\pi)=A \pi+\tilde{\pi} A(I-\pi) .
$$

Now, since $\pi$ is central $U \mid \pi$ is disjoint from $U \mid(I-\pi)$ (i.e., they contain no equivalent subrepresentations) and $\tilde{\pi} A(I-\pi)=0$.

The second identity follows similarly using the inclusion $A^{\prime} H_{\tilde{\pi}} \subset H_{\pi}$, which holds since $A^{\prime} A$ maps $H_{\pi}$ into $H_{\pi}$ for all $A \in I\left(U, U^{*}\right)$. 
Therefore $\pi \oplus \tilde{\pi}$ and $(\pi)^{*} \oplus \tilde{\pi}^{*}$ are central projections. Hence their product $\pi(\tilde{\pi})^{*} \oplus \pi^{*} \tilde{\pi}$ is central, so $\pi(\tilde{\pi})^{*}$ is balanced. Thus $\pi(\tilde{\pi})^{*}=I$ or 0 . If $\pi(\tilde{\pi})^{*}=I$, then $\pi=I$. If $\pi(\tilde{\pi})^{*}=0$, then $\pi+(\tilde{\pi})^{*}$ is a balanced projection and $\pi+(\tilde{\pi})^{*}=I$. But if $\sigma \leqslant \pi$ (i.e., $\sigma \pi=\sigma$ ), then $(\tilde{\sigma})^{*} \leqslant(\tilde{\pi})^{*}$, so that $\sigma+(\tilde{\sigma})^{*}=I$. The latter is impossible unless $\sigma=\pi$. Thus if $\pi \neq I$, then $\pi$ has no smaller central projections. Since $\pi$ was arbitrary this shows that $\pi$ and $I-\pi$ are the only central projections for $U$.

Now, let $\pi$ be a minimal central projection for $U$. By the above lemma $I-\pi$ is also such a projection. By restricting $U$ to the image of $\pi$ we may assume that the only central projection for $U$ is $I$. Hence $U$ is a primary representation so $U$ must be of the form $n U_{0}$ where $U_{0}$ is an irreducible representation and $n \in\{1,2, \ldots, \infty\}$. If one shows that $U_{0}$ is finite dimensional then it will follow that $U$ is finite dimensional since a cyclic representation cannot contain any finite dimensional representation with infinite multiplicity (see [2, §15.5.3]). Thus we can take $U=U_{0}$.

We prove that in the irreducible case the set $\mathcal{C}$ (the domain of $B_{\lambda}$ ) equals $H$. To see this we use the fact that a topologically irreducible representation of a $C^{*}$ algebra is algebraically irreducible (cf. [2, \$2.8.4]). Let $C^{*}(G)$ be the group $C^{*}$ algebra of $G$. Let $U$ (resp., $U^{*}$ ) denote the representation of $C^{*}(G)$ corresponding to $U$ (resp., $\left.U^{*}\right)$. If $f \in C^{*}(G)$, then $\tilde{U}(f) \oplus \tilde{U}^{*}(f) \in \mathcal{Q}$. It follows that $\tilde{U}(f) \varphi \in \mathcal{C}$ for all $f \in C^{*}(G)$. But the set of $\tilde{U}(f) \varphi$ is invariant under $U$, and $U$ is irreducible. Hence $\mathcal{C}=H$ as claimed. Since $B_{\lambda}$ has an adjoint operator, it follows from the closed graph theorem that $B_{\lambda}$ is continuous. As $U$ is irreducible the space $I\left(U, U^{*}\right)$ is at most one dimensional. The mapping $\lambda \rightarrow B_{\lambda}$ is injective from $L^{\prime}$ to $I\left(U, U^{*}\right)$, so $L^{\prime}$ is at most one dimensional. This shows that $L_{+}$has finite codimension in $H$, which implies the finite dimensionality of $H$. Thus the proof of the finite dimensionality of $U$ in general is finished.

Since $U$ is finite dimensional, $B_{\lambda}$ is a continuous operator from $H^{*}$ to $H^{*}$. The mapping $\lambda \rightarrow B_{\lambda}$ is injective. It is also surjective, for if $B \in I\left(U, U^{*}\right)$ then $\lambda=B \varphi \in L^{\prime}$. It is easily seen that $B=B_{\lambda}$. Thus

$$
\operatorname{dim} U=\operatorname{dim} L_{+}+\operatorname{dim} L^{\prime}=\operatorname{dim} L_{+}+\operatorname{dim} I_{a}\left(U, U^{*}\right)
$$

and Theorem 2.1 is proven.

\section{REFERENCES}

1. J. Aczél, Lectures on functional equations and their applications, Academic Press, New York, 1966.

2. J. Dixmier, Les $C^{*}$-algèbres et leurs représentations, deuxième ed., Cahiers Scientifiques, Fasc. XXIX, Gauthier-Villars, Paris, 1969.

3. R. Godement, Les fonctions de type positif et la théorie des groups, Trans. Amer. Math. Soc. 63 (1948), 1-84.

4. M. Hosszu, Some remarks on the cosine functional equation, Publ. Math. Debrecen 16 (1969), 93-98.

5. Pl. Kannappan, The functional equation $f(x y)+f\left(x y^{-1}\right)=2 f(x) f(y)$ for groups, Proc. Amer. Math. Soc. 19 (1968), 69-74. 
6. T. A. O'Connor, A solution of $D^{\prime}$ Alembert's functional equation on a locally compact Abelian group, Aequationes Math. 15 (1977), 235-238.

7. A. L. Rukhin, The families with a universal Bayesian estimator of the transformation parameter, Symposia Mathematica, Istituto Nationale di Alta Mathematica, vol. 21, 1977, pp. 19-26.

Department of Mathematics, Mathematical Sciences Bullding, Purdie University, WEST LAFAYETTE, INDIANA 47907

Departmant of Statistics, Mathematical Sciences Buildng, Purdue University, West LAFAYETTE, INDIANA 47907 